DOE-ER-40757-113

UTEXAS-HEP-98-10

MSUHEP-80715

\title{
High energy photon-neutrino interactions
}

\author{
Ali Abbasabadi ${ }^{1}$, Alberto Devoto ${ }^{2}$, Duane A. Dicus ${ }^{3}$ and Wayne W. Repko ${ }^{4}$ \\ ${ }^{1}$ Department of Physical Sciences, Ferris State University, Big Rapids, Michigan 49307 \\ ${ }^{2}$ Dipartmento di Fisica, Universitá di Cagliari and I.N.F.N., Sezione di Cagliari \\ ${ }^{3}$ Center for Particle Physics and Department of Physics \\ University of Texas, Austin, Texas 78712 \\ ${ }^{4}$ Department of Physics and Astronomy, Michigan State University \\ East Lansing, Michigan 48824
}

(April 30, 2018)

\begin{abstract}
A general decomposition of the amplitudes for the $2 \rightarrow 2$ processes $\gamma \nu \rightarrow \gamma \nu$ and $\gamma \gamma \rightarrow \nu \bar{\nu}$ is obtained using gauge invariance and Bose symmetry. The restrictions implied by this decomposition are investigated for the reaction $\gamma \gamma \rightarrow \nu \bar{\nu}$ by computing the one-loop helicity amplitudes in the standard model. In the center of mass, where $\sqrt{s}=2 \omega$, the cross section grows roughly as $\omega^{6}$ up to the threshold for $W$-boson production, $\sqrt{s}=2 m_{W}$. Astrophysical implications of very high energy photon-neutrino interactions are discussed.
\end{abstract}

13.15.+g, 14.60.Lm,14.70.Bh, 95.30.Cq

Typeset using REVTEX 


\section{INTRODUCTION}

Investigations of the astrophysical importance of the $2 \rightarrow 2$ processes $\gamma \nu \rightarrow \gamma \nu, \gamma \gamma \rightarrow \nu \bar{\nu}$ and $\nu \bar{\nu} \rightarrow \gamma \gamma$ have a long history. These reactions have been examined using the four-Fermi interaction [1], charged vector boson theories [2,3], the standard model [4,5] and modelindependent parameterizations [6]. Because of the vector-axial vector nature of the weak coupling, the cross sections are much smaller than a simple counting of powers of the weak coupling, $G_{F}$, and the fine structure constant, $\alpha$, in the diagrams of Fig. 1 would suggest [7],8]. For massless neutrinos, the cross sections vanish to order $G_{F}^{2} \alpha^{2} \omega^{2}$ [8], and are known to be of order $G_{F}^{4} \omega^{6}$ when $\omega<m_{e}$ [3,5]. This is due to the fact that the scale of the loop integrals for the diagrams in Fig. 1 is set by the $W$-boson mass, $m_{W}$, rather than the electron mass. As a consequence, for center of mass energies $2 \omega$ between $1 \mathrm{keV}$ and $1 \mathrm{MeV}$, the cross sections for $2 \rightarrow 3$ processes such as $\gamma \nu \rightarrow \gamma \gamma \nu$ are larger than the $2 \rightarrow 2$ cross sections [9].

Although of little practical importance in stellar processes where the energy scale is $\lesssim 1 \mathrm{MeV}$, the $2 \rightarrow 2$ processes could be important in very high energy reactions such as $\nu \bar{\nu} \rightarrow \gamma \gamma$ 10], particularly if the $\omega^{6}$ behavior persists for $\omega>m_{e}$. The next Section contains the development of a general decomposition of the elastic amplitude $\mathcal{A}(s, t, u)$ for $\gamma \nu \rightarrow \gamma \nu$. In Section III, we apply this decomposition to the crossed channel $\gamma \gamma \rightarrow \nu \bar{\nu}$ and obtain numerical results for the complete one-loop helicity amplitudes. This is followed by a discussion of our results.

\section{GENERAL DECOMPOSITION OF THE $\gamma \nu$ ELASTIC AMPLITUDE}

The amplitude for the process $\gamma \nu \rightarrow \gamma \nu$ can be expressed as

$$
\mathcal{A}(s, t, u)=\bar{u}\left(p_{2}\right) \gamma_{\mu}\left(1+\gamma_{5}\right) u\left(p_{1}\right) \mathcal{M}_{\mu \alpha \beta}\left(\varepsilon_{1}\right)_{\alpha}\left(\varepsilon_{2}^{*}\right)_{\beta}
$$

where $\mathcal{M}_{\mu \alpha \beta}$ is a sum of gauge invariant, Bose symmetric tensors constructed from the neutrino momenta $p_{1}, p_{2}$ and the photon momenta $k_{1}, k_{2}$. Construction of these tensors is aided by the fact that, for massless neutrinos, we have

$$
\begin{aligned}
\left(p_{1}\right)_{\mu} \bar{u}\left(p_{2}\right) \gamma_{\mu}\left(1+\gamma_{5}\right) u\left(p_{1}\right) & =0, \\
\left(p_{2}\right)_{\mu} \bar{u}\left(p_{2}\right) \gamma_{\mu}\left(1+\gamma_{5}\right) u\left(p_{1}\right) & =0, \\
\left(k_{1}-k_{2}\right)_{\mu} \bar{u}\left(p_{2}\right) \gamma_{\mu}\left(1+\gamma_{5}\right) u\left(p_{1}\right) & =0 .
\end{aligned}
$$

This suggests the use of the combinations

$$
\begin{aligned}
p & =p_{1}+p_{2}, \\
k_{+} & =k_{1}+k_{2}, \\
k_{-} & =k_{1}-k_{2} .
\end{aligned}
$$

From the above, of $p_{\alpha}, p_{\beta}$ and $p_{\mu}$, only $p_{\alpha}$ and $p_{\beta}$ will result in non-vanishing contributions. Thus, the vector-vector and axial vector-axial vector contributions to $\mathcal{M}_{\mu \alpha \beta}$ will consist of combinations of $p_{\alpha}, p_{\beta},\left(k_{+}\right)_{\mu},\left(k_{2}\right)_{\alpha}$ and $\left(k_{1}\right)_{\beta}$ as well as $\delta_{\mu \alpha}, \delta_{\mu \beta}$ and $\delta_{\alpha \beta}$.

The gauge invariant combinations of $p_{\alpha}$ and $p_{\beta}$ are 


$$
p_{\alpha}-\frac{k_{+} \cdot p}{k_{+}^{2}}\left(k_{2}\right)_{\alpha} \text { and } p_{\beta}-\frac{k_{+} \cdot p}{k_{+}^{2}}\left(k_{1}\right)_{\beta},
$$

and we note that $k_{+} \cdot p=2 k_{1} \cdot p=2 k_{2} \cdot p$ and $k_{+}^{2}=2 k_{1} \cdot k_{2}$. Gauge invariant second rank tensors involving $\delta_{\mu \alpha}$ and $\delta_{\mu \beta}$ are

$$
\begin{gathered}
\left(k_{1} \cdot k_{2} \delta_{\mu \alpha}-\left(k_{1}\right)_{\mu}\left(k_{2}\right)_{\alpha}\right) \text { and }\left(k_{1} \cdot k_{2} \delta_{\mu \beta}-\left(k_{2}\right)_{\mu}\left(k_{1}\right)_{\beta}\right), \\
\left(k_{1} \cdot p \delta_{\mu \alpha}-\left(k_{1}\right)_{\mu} p_{\alpha}\right) \text { and }\left(k_{2} \cdot p \delta_{\mu \beta}-\left(k_{2}\right)_{\mu} p_{\beta}\right),
\end{gathered}
$$

and $\delta_{\alpha \beta}$ appears in the form

$$
\left(k_{1} \cdot k_{2} \delta_{\alpha \beta}-\left(k_{2}\right)_{\alpha}\left(k_{1}\right)_{\beta}\right) .
$$

Gauge invariant third rank tensors can then be constructed from products of $\left(k_{+}\right)_{\mu}$, the vectors in Eq. (6), and the second rank tensors in Eqs. (강). In doing so, it should be noted that combinations such as

$$
\begin{array}{r}
\left(k_{1} \cdot k_{2} \delta_{\mu \alpha}-\left(k_{1}\right)_{\mu}\left(k_{2}\right)_{\alpha}\right)\left(p_{\beta}-\frac{k_{+} \cdot p}{k_{+}^{2}}\left(k_{1}\right)_{\beta}\right), \\
\left(k_{1} \cdot p \delta_{\mu \alpha}-\left(k_{1}\right)_{\mu} p_{\alpha}\right)\left(p_{\beta}-\frac{k_{+} \cdot p}{k_{+}^{2}}\left(k_{1}\right)_{\beta}\right), \\
\left(k_{+}\right)_{\mu}\left(p_{\alpha}-\frac{k_{+} \cdot p}{k_{+}^{2}}\left(k_{2}\right)_{\alpha}\right)\left(p_{\beta}-\frac{k_{+} \cdot p}{k_{+}^{2}}\left(k_{1}\right)_{\beta}\right),
\end{array}
$$

are not linearly independent since

$$
\frac{k_{+} \cdot p}{k_{1} \cdot k_{2}}(10)-(12)=2(11) \text {. }
$$

As a consequence, the tensors of Eq. (8) can be omitted when constructing a complete set of gauge invariant third rank tensors.

The requirement of Bose symmetry can easily be included if one constructs gauge invariant tensors that have a definite symmetry under the exchanges $k_{1} \rightarrow-k_{2}, k_{2} \rightarrow-k_{1}$ and $\alpha \leftrightarrow \beta$. The vector $k_{+} \rightarrow-k_{+}$under these exchanges. It is possible to construct four linearly independent, gauge invariant third rank tensors with definite symmetry under the exchange of the photons. They can be chosen to be

$$
\begin{aligned}
T_{\mu \alpha \beta}^{(1)}= & \left(k_{1} \cdot k_{2} \delta_{\mu \alpha}-\left(k_{1}\right)_{\mu}\left(k_{2}\right)_{\alpha}\right)\left(p_{\beta}-\frac{k_{+} \cdot p}{k_{+}^{2}}\left(k_{1}\right)_{\beta}\right)+ \\
& \left(k_{1} \cdot k_{2} \delta_{\mu \beta}-\left(k_{2}\right)_{\mu}\left(k_{1}\right)_{\beta}\right)\left(p_{\alpha}-\frac{k_{+} \cdot p}{k_{+}^{2}}\left(k_{2}\right)_{\alpha}\right), \\
T_{\mu \alpha \beta}^{(2)}= & \left(k_{1} \cdot k_{2} \delta_{\mu \alpha}-\left(k_{1}\right)_{\mu}\left(k_{2}\right)_{\alpha}\right)\left(p_{\beta}-\frac{k_{+} \cdot p}{k_{+}^{2}}\left(k_{1}\right)_{\beta}\right)- \\
& \left(k_{1} \cdot k_{2} \delta_{\mu \beta}-\left(k_{2}\right)_{\mu}\left(k_{1}\right)_{\beta}\right)\left(p_{\alpha}-\frac{k_{+} \cdot p}{k_{+}^{2}}\left(k_{2}\right)_{\alpha}\right), \\
T_{\mu \alpha \beta}^{(3)}= & \left(k_{+}\right)_{\mu}\left(k_{1} \cdot k_{2} \delta_{\alpha \beta}-\left(k_{2}\right)_{\alpha}\left(k_{1}\right)_{\beta}\right), \\
T_{\mu \alpha \beta}^{(4)}= & \left(k_{+}\right)_{\mu}\left(p_{\alpha}-\frac{k_{+} \cdot p}{k_{+}^{2}}\left(k_{2}\right)_{\alpha}\right)\left(p_{\beta}-\frac{k_{+} \cdot p}{k_{+}^{2}}\left(k_{1}\right)_{\beta}\right) .
\end{aligned}
$$


It can be seen that $T_{\mu \alpha \beta}^{(1)}$ is symmetric under the exchange of photons, while $T_{\mu \alpha \beta}^{(2)}, T_{\mu \alpha \beta}^{(3)}$ and $T_{\mu \alpha \beta}^{(4)}$ are antisymmetric.

To obtain tensors which correspond to the vector-axial vector interference terms, one can use a set of independent momenta together with $\varepsilon_{\alpha \beta \lambda \rho}$ to form gauge invariant tensors with definite symmetry under the exchange of the photons. When this is done, one finds two tensors with the appropriate properties. They have the form

$$
\begin{aligned}
T_{\mu \alpha \beta}^{(5)}= & \left(k_{+}\right)_{\mu} \varepsilon_{\alpha \beta \lambda \rho}\left(k_{+}\right)_{\lambda}\left(k_{-}\right)_{\rho}, \\
T_{\mu \alpha \beta}^{(6)}= & \left(k_{+}\right)_{\mu} \varepsilon_{\alpha \beta \lambda \rho}\left(k_{+}\right)_{\lambda} p_{\rho}-k_{+}^{2} \varepsilon_{\mu \alpha \beta \lambda} p_{\lambda}-\delta_{\alpha \beta} \varepsilon_{\mu \nu \lambda \rho}\left(k_{+}\right)_{\nu}\left(k_{-}\right)_{\lambda} p_{\rho} \\
& +p_{\alpha} \varepsilon_{\mu \beta \lambda \rho}\left(k_{+}\right)_{\lambda}\left(k_{-}\right)_{\rho}+p_{\beta} \varepsilon_{\mu \alpha \lambda \rho}\left(k_{+}\right)_{\lambda}\left(k_{-}\right)_{\rho} .
\end{aligned}
$$

The gauge invariance of Eq. (19) can be verified using the identity

$$
k_{\mu} \varepsilon_{\alpha \beta \lambda \rho}+k_{\alpha} \varepsilon_{\beta \lambda \rho \mu}+k_{\beta} \varepsilon_{\lambda \rho \mu \alpha}+k_{\lambda} \varepsilon_{\rho \mu \alpha \beta}+k_{\rho} \varepsilon_{\mu \alpha \beta \lambda}=0
$$

which holds for any vector $k$. Using this identity, the factor $\xi_{\mu}=\bar{u}\left(p_{2}\right) \gamma_{\mu}\left(1+\gamma_{5}\right) u\left(p_{1}\right)$ in Eq. (1) can always be rewritten so that it is contracted with $\varepsilon_{\mu \nu \alpha \beta}$ instead of with $\left(k_{+}\right)_{\mu}$. The relation

$$
-p_{1} \cdot p_{2} \xi_{\mu}=\varepsilon_{\mu \nu \alpha \beta} \xi_{\nu}\left(p_{1}\right)_{\alpha}\left(p_{2}\right)_{\beta},
$$

then enables us to express any tensor containing an $\varepsilon_{\mu \nu \alpha \beta}$ as a sum of scalar products. These are simply combinations of the $T_{\mu \alpha \beta}^{(i)}$ of Eqs. (14 [17). Explicitly, we find [11]

$$
\begin{aligned}
& T_{\mu \alpha \beta}^{(5)} \propto T_{\mu \alpha \beta}^{(2)}, \\
& T_{\mu \alpha \beta}^{(6)} \propto\left(T_{\mu \alpha \beta}^{(3)}+\frac{p \cdot k_{+}}{k_{+}^{2}} T_{\mu \alpha \beta}^{(1)}-T_{\mu \alpha \beta}^{(4)}\right) .
\end{aligned}
$$

Consequently, the $T_{\mu \alpha \beta}^{(i)}, i=1, \cdots, 4$, are sufficient to parameterize the $\gamma \nu$ elastic amplitude.

The tensor $\mathcal{M}_{\mu \alpha \beta}$ can thus be written

$$
\mathcal{M}_{\mu \alpha \beta}=\mathcal{M}_{1}(s, t, u) T_{\mu \alpha \beta}^{(1)}+\mathcal{M}_{2}(s, t, u) T_{\mu \alpha \beta}^{(2)}+\mathcal{M}_{3}(s, t, u) T_{\mu \alpha \beta}^{(3)}+\mathcal{M}_{4}(s, t, u) T_{\mu \alpha \beta}^{(4)},
$$

where Bose symmetry requires

$$
\begin{aligned}
& \mathcal{M}_{1}(s, t, u)=\mathcal{M}_{1}(u, t, s), \\
& \mathcal{M}_{j}(s, t, u)=-\mathcal{M}_{j}(u, t, s), j=2,3,4 .
\end{aligned}
$$

Using the center of mass frame, and the explicit forms of all the vectors, we can obtain the following helicity basis

$$
\begin{aligned}
& T_{\mu \alpha \beta}^{(1)} \xi_{\mu}\left(\varepsilon_{1}\right)_{\alpha}\left(\varepsilon_{2}^{*}\right)_{\beta}=-s \cos (\theta / 2)\left(t\left(\lambda_{1}+\lambda_{2}+2 \lambda_{1} \lambda_{2}\right)+4 s \lambda_{1} \lambda_{2}\right), \\
& T_{\mu \alpha \beta}^{(2)} \xi_{\mu}\left(\varepsilon_{1}\right)_{\alpha}\left(\varepsilon_{2}^{*}\right)_{\beta}=s t \cos (\theta / 2)\left(\lambda_{1}-\lambda_{2}\right) \\
& T_{\mu \alpha \beta}^{(3)} \xi_{\mu}\left(\varepsilon_{1}\right)_{\alpha}\left(\varepsilon_{2}^{*}\right)_{\beta}=s t \cos (\theta / 2)\left(1-\lambda_{1} \lambda_{2}\right) \\
& T_{\mu \alpha \beta}^{(4)} \xi_{\mu}\left(\varepsilon_{1}\right)_{\alpha}\left(\varepsilon_{2}^{*}\right)_{\beta}=-8 \frac{s^{2} u}{t} \cos (\theta / 2) \lambda_{1} \lambda_{2}
\end{aligned}
$$


where $\theta$ is the angle between the incoming and outgoing neutrinos, $\lambda_{1}$ is the helicity of the initial photon, $\lambda_{2}$ is the helicity of the final photon and it is unnecessary to specify the neutrino helicities since they have a definite handedness. Notice that the first, third and fourth of these amplitudes are symmetric under the exchange of $\lambda_{1}$ and $\lambda_{2}$, while the second is antisymmetric under this exchange. Since time reversal invariance implies that the amplitude should be symmetric under the exchange of initial and final helicities, the second amplitude is T-violating. There are thus three helicity amplitudes if we require time reversal symmetry. This is consistent with simple helicity counting arguments.

\section{THE CHANNEL $\gamma \gamma \rightarrow \nu \bar{\nu}$}

The results of Section [I] can be expressed in the crossed channel $\gamma \gamma \rightarrow \nu \bar{\nu}$ using the relations $p_{1} \rightarrow-p_{1}$ and $k_{2} \rightarrow-k_{2}$, which imply the interchange $s \leftrightarrow t$. The combinations $k_{+}$and $p$ become

$$
\begin{aligned}
k_{+} & =k_{1}+k_{2} \rightarrow k_{1}-k_{2} \equiv k_{-} \\
p & =p_{1}+p_{2} \rightarrow-p_{1}+p_{2} \equiv-p
\end{aligned}
$$

These transformations allow us to write the cross channel counterparts of Eqs. (14 17) as

$$
\begin{aligned}
\tilde{T}_{\mu \alpha \beta}^{(1)}= & \left(k_{1} \cdot k_{2} \delta_{\mu \alpha}-\left(k_{1}\right)_{\mu}\left(k_{2}\right)_{\alpha}\right)\left(p_{\beta}-\frac{k_{-} \cdot p}{k_{-}^{2}}\left(k_{1}\right)_{\beta}\right)+ \\
& \left(k_{1} \cdot k_{2} \delta_{\mu \beta}-\left(k_{2}\right)_{\mu}\left(k_{1}\right)_{\beta}\right)\left(p_{\alpha}+\frac{k_{-} \cdot p}{k_{-}^{2}}\left(k_{2}\right)_{\alpha}\right), \\
\tilde{T}_{\mu \alpha \beta}^{(2)}= & \left(k_{1} \cdot k_{2} \delta_{\mu \alpha}-\left(k_{1}\right)_{\mu}\left(k_{2}\right)_{\alpha}\right)\left(p_{\beta}-\frac{k_{-} \cdot p}{k_{-}^{2}}\left(k_{1}\right)_{\beta}\right)- \\
& \left(k_{1} \cdot k_{2} \delta_{\mu \beta}-\left(k_{2}\right)_{\mu}\left(k_{1}\right)_{\beta}\right)\left(p_{\alpha}+\frac{k_{-} \cdot p}{k_{-}^{2}}\left(k_{2}\right)_{\alpha}\right), \\
\tilde{T}_{\mu \alpha \beta}^{(3)}= & -\left(k_{-}\right)_{\mu}\left(k_{1} \cdot k_{2} \delta_{\alpha \beta}-\left(k_{2}\right)_{\alpha}\left(k_{1}\right)_{\beta}\right), \\
\tilde{T}_{\mu \alpha \beta}^{(4)}= & \left(k_{-}\right)_{\mu}\left(p_{\alpha}+\frac{k_{-} \cdot p}{k_{-}^{2}}\left(k_{2}\right)_{\alpha}\right)\left(p_{\beta}-\frac{k_{-} \cdot p}{k_{-}^{2}}\left(k_{1}\right)_{\beta}\right) .
\end{aligned}
$$

The amplitude can be expanded as

$$
\tilde{\mathcal{M}}_{\mu \alpha \beta}=\mathcal{M}_{1}(t, s, u) \tilde{T}_{\mu \alpha \beta}^{(1)}+\mathcal{M}_{2}(t, s, u) \tilde{T}_{\mu \alpha \beta}^{(2)}+\mathcal{M}_{3}(t, s, u) \tilde{T}_{\mu \alpha \beta}^{(3)}+\mathcal{M}_{4}(t, s, u) \tilde{T}_{\mu \alpha \beta}^{(4)},
$$

where

$$
\begin{aligned}
& \mathcal{M}_{1}(t, s, u)=\mathcal{M}_{1}(u, s, t), \\
& \mathcal{M}_{j}(t, s, u)=-\mathcal{M}_{j}(u, s, t), j=2,3,4
\end{aligned}
$$

In this case, the center of mass helicity basis is 


$$
\begin{aligned}
& \tilde{T}_{\mu \alpha \beta}^{(1)} \tilde{\xi}_{\mu}\left(\varepsilon_{1}\right)_{\alpha}\left(\varepsilon_{2}\right)_{\beta}=\frac{1}{2} s \sin \theta\left(s\left(\lambda_{1}-\lambda_{2}+2 \lambda_{1} \lambda_{2}\right)+4 t \lambda_{1} \lambda_{2}\right), \\
& \tilde{T}_{\mu \alpha \beta}^{(2)} \tilde{\xi}_{\mu}\left(\varepsilon_{1}\right)_{\alpha}\left(\varepsilon_{2}\right)_{\beta}=-\frac{1}{2} s^{2} \sin \theta\left(\lambda_{1}+\lambda_{2}\right), \\
& \tilde{T}_{\mu \alpha \beta}^{(3)} \tilde{\xi}_{\mu}\left(\varepsilon_{1}\right)_{\alpha}\left(\varepsilon_{2}\right)_{\beta}=\frac{1}{2} s^{2} \sin \theta\left(1+\lambda_{1} \lambda_{2}\right), \\
& \tilde{T}_{\mu \alpha \beta}^{(4)} \tilde{\xi}_{\mu}\left(\varepsilon_{1}\right)_{\alpha}\left(\varepsilon_{2}\right)_{\beta}=4 t u \sin \theta \lambda_{1} \lambda_{2} .
\end{aligned}
$$

Here, $\tilde{\xi}_{\mu}=\bar{u}\left(p_{1}\right) \gamma_{\mu}\left(1+\gamma_{5}\right) v\left(p_{2}\right)$ and $\theta$ is the angle between photon 1 , which is in the $z$ direction, and the outgoing neutrino. Using the helicity basis, we can express the helicity amplitudes $\tilde{\mathcal{A}}_{\lambda_{1} \lambda_{2}}(s, z)$, where $z=\cos \theta$, as

$$
\begin{aligned}
& \tilde{\mathcal{A}}_{++}(s, z)=\sin \theta\left[s(t-u) \mathcal{M}_{1}(t, s, u)+s^{2} \mathcal{M}_{3}(t, s, u)+4 t u \mathcal{M}_{4}(t, s, u)\right], \\
& \tilde{\mathcal{A}}_{+-}(s, z)=\sin \theta\left[-2 s t \mathcal{M}_{1}(t, s, u)-4 t u \mathcal{M}_{4}(t, s, u)\right], \\
& \tilde{\mathcal{A}}_{-+}(s, z)=\sin \theta\left[2 s u \mathcal{M}_{1}(t, s, u)-4 t u \mathcal{M}_{4}(t, s, u)\right], \\
& \tilde{\mathcal{A}}_{--}(s, z)=\sin \theta\left[s(t-u) \mathcal{M}_{1}(t, s, u)+s^{2} \mathcal{M}_{3}(t, s, u)+4 t u \mathcal{M}_{4}(t, s, u)\right],
\end{aligned}
$$

where we have assumed time reversal symmetry and omitted $\mathcal{M}_{2}(t, s, u)$. From Eqs. (43) and (46), is is clear that $\tilde{\mathcal{A}}_{++}(s, z)=\tilde{\mathcal{A}}_{--}(s, z)$. Moreover, since $t=-\frac{1}{2} s(1-z)$ and $u=-\frac{1}{2} s(1+z)$, the symmetries of Eqs. (37) and (38) imply

$$
\tilde{\mathcal{A}}_{++}(s, z)=-\tilde{\mathcal{A}}_{++}(s,-z) \text {. }
$$

Similarly, we find that the helicity flip amplitudes satisfy

$$
\tilde{\mathcal{A}}_{+-}(s, z)=-\tilde{\mathcal{A}}_{-+}(s,-z) .
$$

To explore these general results, we calculated the standard model diagrams of Fig. 1 using a nonlinear $R_{\xi}$ gauge such that the coupling between the photon, the $W$-boson and the Goldstone boson vanishes [12,13,5]. These diagrams can be decomposed in terms of scalar two-point, three-point and four-point functions, which are expressible in terms of dilogarithms [14]. The expressions for the helicity amplitudes in terms of dilogarithms were evaluated numerically and the results checked against FORTRAN codes developed for oneloop integrals 115,16. In doing so, we assumed that $s>m_{\ell}^{2}$, where $m_{\ell}$ is the lepton mass, and showed that the dependence on $\ln \left(m_{\ell}^{2}\right)$ or $\ln ^{2}\left(m_{\ell}^{2}\right)$ from individual diagrams actually cancels.

The results for the helicity amplitudes are shown in Figs.(2) and (3), where the cross sections for various helicities are plotted for $\sqrt{s}=20 \mathrm{GeV}$ and $\sqrt{s}=200 \mathrm{GeV}$ using

$$
\frac{d \sigma_{\lambda_{1} \lambda_{2}}}{d z}=\frac{1}{32 \pi s}\left|\tilde{\mathcal{A}}_{\lambda_{1} \lambda_{2}}\right|^{2}
$$

These figures clearly illustrate the symmetries of Eqs. (47) and (48), particularly the vanishing of the non-flip amplitudes at $z=0$. The total cross section for unpolarized photons is given by 


$$
\sigma_{\gamma \gamma \rightarrow \nu \bar{\nu}}=\frac{1}{64 \pi s} \int_{-1}^{1} d z\left[\left|\tilde{\mathcal{A}}_{++}(s, z)\right|^{2}+\left|\tilde{\mathcal{A}}_{+-}(s, z)\right|^{2}\right]
$$

and is plotted in Fig. (4). This figure illustrates the roughly $s^{3}$ behavior of the total cross section up to the threshold for $W$ pair production. Also shown in dashes is the helicity non-flip contribution to the cross section, which can be seen to be much smaller than the helicity flip contribution. This feature does not appear to be related to any symmetry, but it is reminiscent of the low energy case, where the non-flip amplitudes vanish [5].

\section{DISCUSSION AND CONCLUSIONS}

As mentioned in the Introduction, the low energy $2 \rightarrow 2$ photon-neutrino cross sections are much smaller than the corresponding $2 \rightarrow 3$ cross sections for center of mass energies between $1 \mathrm{keV}$ and $1 \mathrm{MeV}$. Recently, the $2 \rightarrow 3$ cross sections have been computed for a range of center of mass energies from well below $2 m_{e}$, where the cross sections vary as $\omega^{10}$, to well above $2 m_{e}$, but less than $m_{W}$ [17]. For $m_{e}<<\omega<<m_{W}$, the only scale in the $2 \rightarrow 3$ processes is $m_{W}$ or, equivalently, $G_{F}$. From dimensional considerations, the center of mass cross section must behave as $G_{F}^{2} \omega^{2}$ in this range of energy. Explicitly, it is found that $\sigma_{\gamma \gamma \rightarrow \nu \bar{\nu} \gamma}$ can be expressed as

$$
\sigma_{\gamma \gamma \rightarrow \nu \bar{\nu} \gamma}=5.68 \times 10^{-14}\left(\frac{\omega}{m_{e}}\right)^{2} \mathrm{fb} .
$$

For the $2 \rightarrow 2$ process $\gamma \gamma \rightarrow \nu \bar{\nu}$, we expect an $\omega^{6}$ behavior, and a fit to the data in Fig. (4) yields

$$
\sigma_{\gamma \gamma \rightarrow \nu \bar{\nu}}=4.0 \times 10^{-31}\left(\frac{\omega}{m_{e}}\right)^{6} \mathrm{fb}
$$

These expressions are equal for $\omega=1.94 \times 10^{4} m_{e}$ or $\sqrt{s} \sim 20 \mathrm{GeV}$. Thus, for sufficiently high energies, the $2 \rightarrow 2$ process dominates the $2 \rightarrow 3$ process.

To assess the importance of very high energy photon-neutrino interactions in processes of interest in cosmology, consider the scattering of high energy neutrinos from the cosmic neutrino background. This process has recently been studied by assuming that the neutrino collision produces a $Z$ at resonance whose decay chain contains photons and protons which could account for the flux of $>10^{20} \mathrm{eV}$ cosmic rays [10]. For the processes considered here, the $\nu \bar{\nu}$ collision would directly produce high energy photons. The cross section for $\nu \bar{\nu} \rightarrow \gamma \gamma$ can be obtained from Eq. (52) by supplying a factor of 2. Assuming the relic neutrinos have a small mass [10:18], the cross section in the rest frame of the target is

$$
\sigma_{\nu \bar{\nu} \rightarrow \gamma \gamma}=5.6 \times 10^{-42}\left(\tilde{m}_{\nu} \tilde{E}_{\nu}\right)^{3} \mathrm{~cm}^{2},
$$

where $\tilde{m}_{\nu}$ is $m_{\nu} / 1 \mathrm{eV}$ and $\tilde{E}_{\nu}$ is $E_{\nu} / 10^{21} \mathrm{eV}$. The interaction rate on the cosmic neutrino background is $\sigma_{\nu \bar{\nu} \rightarrow \gamma \gamma} n_{\nu} c$, where $n_{\nu}$ is the relic neutrino density. If this is multiplied by $t_{0}$, the age of the universe, the condition that at least one interaction occur is

$$
\sigma_{\nu \bar{\nu} \rightarrow \gamma \gamma} n_{\nu} c t_{0}=1
$$


or

$$
\tilde{m}_{\nu} \tilde{E}_{\nu}=\frac{5.6 \times 10^{13} \mathrm{~cm}^{-2 / 3}}{\left(n_{\nu} c t_{0}\right)^{1 / 3}} .
$$

Taking $n_{\nu}=56 \mathrm{~cm}^{-3}$ and $t_{0}=15 \times 10^{9}$ years, $\tilde{m}_{\nu} \tilde{E}_{\nu}=6.07 \times 10^{3}$, which translates into $\sqrt{s}=3.5 \mathrm{TeV}$. Even if there were sources of such high energy neutrinos (which seems unlikely), this is well above the region for which the $s^{3}$ behavior is valid. In fact, the relation $\left(n_{\nu} c t_{0}\right)^{-1}=1.26 \times 10^{-30} \mathrm{~cm}^{2}$ together with Fig. (4) show that $\sigma_{\nu \bar{\nu} \rightarrow \gamma \gamma}$ is not large enough for any value of $s$ to attenuate a high energy neutrino flux given the present values of $n_{\nu}$ and the age of the universe.

The temperature at which the reaction $\nu \bar{\nu} \rightarrow \gamma \gamma$ ceases to occur can be determined from the reaction rate per unit volume

$$
\rho=\frac{1}{(2 \pi)^{6}} \int \frac{d^{3} p_{1}}{e^{E_{1} / T}+1} \int \frac{d^{3} p_{2}}{e^{E_{2} / T}+1} \sigma|\vec{v}|,
$$

where $\vec{p}_{1}$ and $\vec{p}_{2}$ are the neutrino and antineutrino momenta, $E_{1}$ and $E_{2}$ their energies, $|\vec{v}|$ is the flux and $T$ the temperature. Using the invariance of $\sigma E_{1} E_{2}|\vec{v}|$, the relationship between $\sigma|\vec{v}|$ in the center of mass frame and any other frame is

$$
\sigma|\vec{v}|=\sigma_{C M} \frac{2 E_{\mathrm{CM}}^{2}}{E_{1} E_{2}}
$$

If the angle between $\vec{p}_{1}$ and $\vec{p}_{2}$ is $\theta_{12}$, Eq. (57) gives

$$
\sigma_{\nu \bar{\nu} \rightarrow \gamma \gamma}|\vec{v}|=1.6 \times 10^{-30} \frac{E_{1}^{3} E_{2}^{3}}{m_{e}^{6}} \sin ^{8}\left(\theta_{12} / 2\right) \mathrm{fb} .
$$

The integration is straightforward and Eq. (56) gives

$$
\rho_{\nu \bar{\nu} \rightarrow \gamma \gamma}=\frac{1.6 \times 10^{-30} \mathrm{fb}}{5 \pi^{4}}\left(\frac{31}{32} \Gamma(6) \zeta(6)\right)^{2} \frac{T^{12}}{m_{e}^{6}},
$$

where $\zeta(x)$ is the Riemann zeta function. The interaction rate $R_{\nu \bar{\nu} \rightarrow \gamma \gamma}$ can be obtained by dividing $\rho_{\nu \bar{\nu} \rightarrow \gamma \gamma}$ by the neutrino density $n_{\nu}=3 \zeta(3) T^{3} / 4 \pi^{2}$, and we find

$$
R_{\nu \bar{\nu} \rightarrow \gamma \gamma}=7.3 \times 10^{-24} T_{10}^{9} \mathrm{~s}^{-1},
$$

with $T_{10}$ denoting $T / 10^{10} \mathrm{~K}$. Expressing the age of the universe as $t=2 T_{10}^{-2} \mathrm{~s}$, the condition for at least one interaction, $R_{\nu \bar{\nu} \rightarrow \gamma \gamma} t \sim 1$ gives $T \sim 1.6 \mathrm{GeV}$, which is within the region of validity of the $s^{3}$ behavior.

\section{ACKNOWLEDGMENTS}

One of us (A. D.) wishes to thank the Department of Physics and Astronomy of Michigan State University for its support and hospitality. This research was supported in part by the U.S. Department of Energy under Contract No. DE-FG013-93ER40757 and in part by the National Science Foundation under Grants No. PHY-93-07980 and PHY-98-02439. 


\section{REFERENCES}

[1] H.-Y. Chiu and P. Morrison, Phys. Rev. Lett. 5, 573 (1960).

[2] M. J. Levine, Nuovo Cimento 48A, 67 (1967).

[3] L. F. Landovitz and W. M. Schreiber, Nuovo Cimento 2A, 359 (1971).

[4] V. K. Cung and M. Yoshimura, Nuovo Cimento 29A, 557 (1975).

[5] D. A. Dicus and W. W. Repko, Phys. Rev. D 48, 5106 (1993).

[6] J. Liu, Phys. Rev. D 44, 2879 (1991).

[7] C. N. Yang, Phys. Rev. 77, 242 (1950); L. D. Landau, Sov. Phys. Doklady 60, 207 (1948).

[8] M. Gell-Mann, Phys. Rev. Lett. 6, 70 (1961).

[9] D. A. Dicus and W. W. Repko, Phys. Rev. Lett. 79, 569 (1997). The channel $\gamma \gamma \rightarrow \nu \bar{\nu} \gamma$ is discussed in: N. Van Hieu and E. P. Shabalin, Sov. Phys. JETP 17, 681 (1963). See also: A. Abada, J. Matias and R. Pittau, hep-ph/9806383 (1998).

[10] E. Waxman, astro-ph/9804023 (1998).

[11] This is most easily shown using Eqs. (27-30) and the corresponding expressions for $T_{\mu \alpha \beta}^{(5)}$ and $T_{\mu \alpha \beta}^{(6)}$.

[12] M. B. Gavela, G. Girardi, C. Malleville, and P. Sorba, Nucl. Phys. B193, 257 (1981);

M. Bace and N. D. Hari Dass, Ann. of Phys. 94, 349 (1975).

[13] J. F. Nieves, P. B. Pal, and D. G. Unger, Phys. Rev. D28, 908 (1983).

[14] G. Passarino and M. Veltman, Nucl. Phys. B160, 151 (1979).

[15] C. Kao and D. A. Dicus, LOOP, a FORTRAN program for evaluating loop integrals based on the results in: G. 't Hooft and M. Veltman, Nucl. Phys. B153, 365 (1979), and Ref. [14].

[16] G. J. van Oldenborgh, NIKHEF-H/90-15, (1990).

[17] D. A. Dicus, C. Kao and W. W. Repko, hep-ph/9806499 (1998) (submitted to Phys. Rev.).

[18] The corrections to the $s^{3}$ behavior of Eq. (52) due to a small neutrino mass are of order $m_{\nu}^{2} / s$, which is negligible. 


\section{FIGURES}
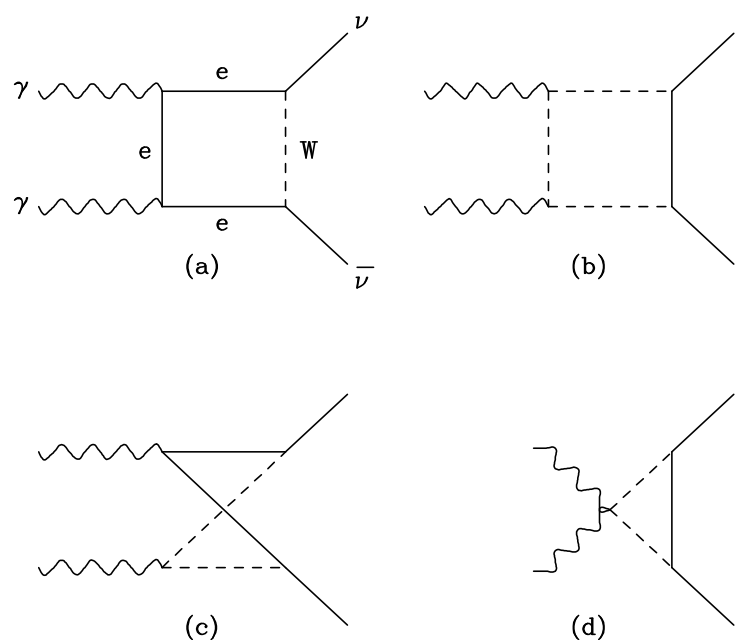

FIG. 1. Diagrams for the process $\gamma \gamma \rightarrow \nu_{e} \bar{\nu}_{e}$. For each of (a), (b), (c) there is also a diagram with the photons interchanged.

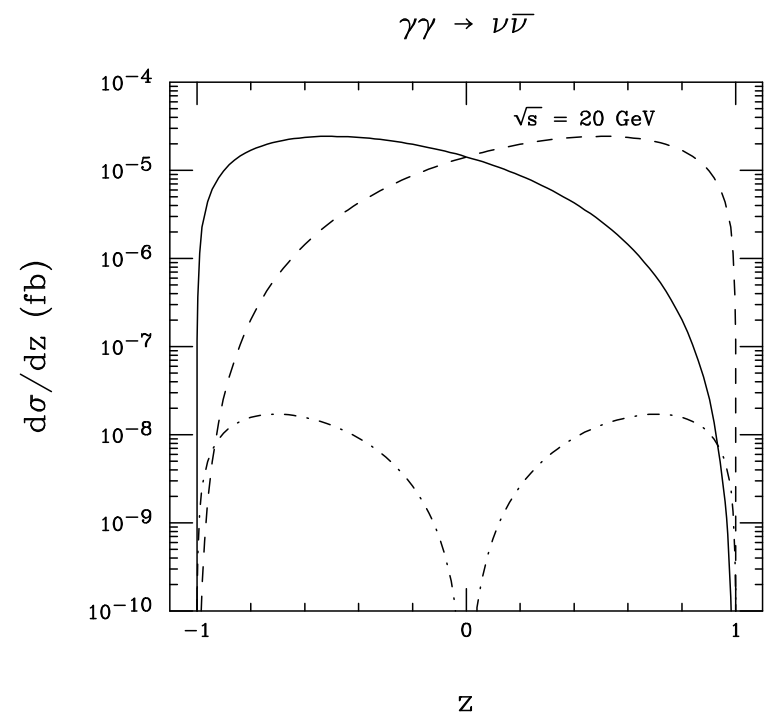

FIG. 2. The helicity dependent differential cross sections for $\gamma \gamma \rightarrow \nu \bar{\nu}$ are shown for $\sqrt{s}=20$ $\mathrm{GeV}$. The solid line is $d \sigma_{+-} / d z$, the dashed line is $d \sigma_{-+} / d z$ and the dot-dashed line is $d \sigma_{++} / d z$. 


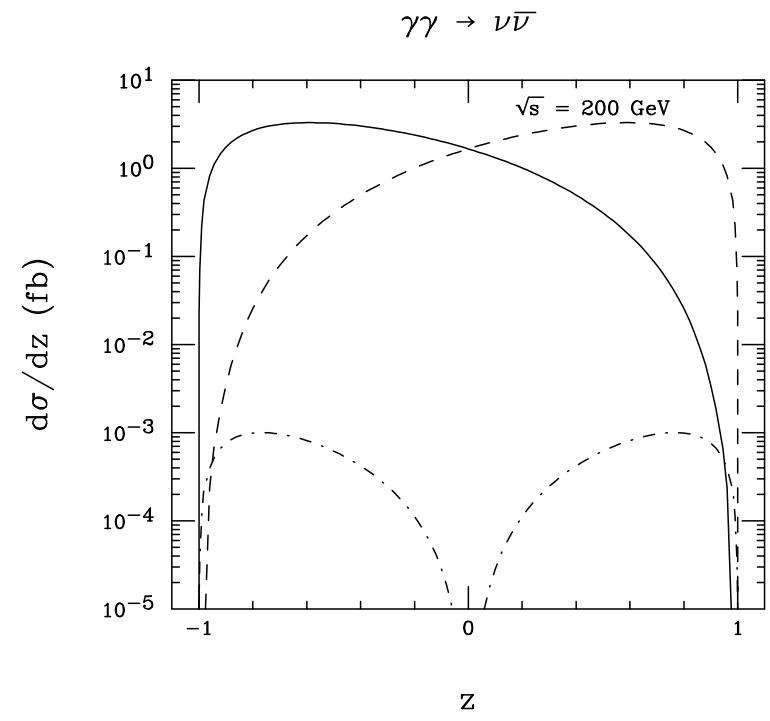

FIG. 3. Same as Fig. (2) with $\sqrt{s}=200 \mathrm{GeV}$.
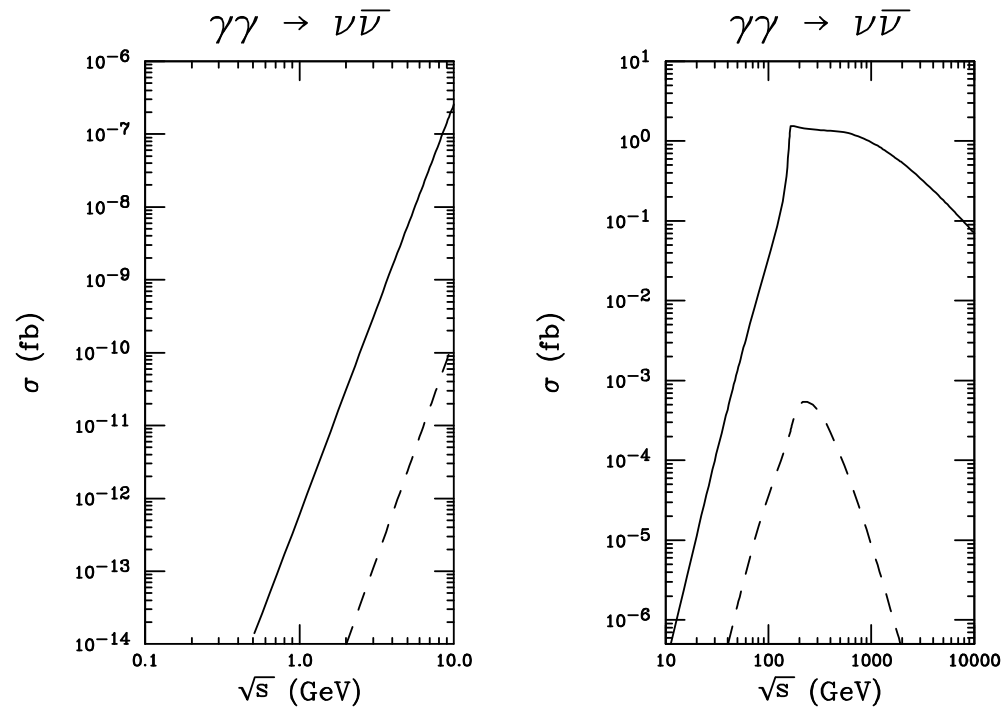

FIG. 4. The solid line is the total cross section $\sigma_{\gamma \gamma \rightarrow \nu \bar{\nu}}$ and the dashed line is the contribution from the helicity non-flip amplitudes. 